\title{
Ages and metallicities of faint red galaxies in the Shapley Supercluster
}

\author{
Russell J. Smith ${ }^{1}$, John R. Lucey ${ }^{1}$ and Michael J. Hudson ${ }^{2}$ \\ ${ }^{1}$ Department of Physics, University of Durham, Durham, United Kingdom \\ ${ }^{2}$ Department of Physics and Astronomy, University of Waterloo, Waterloo, ON, Canada
}

\begin{abstract}
We present results on the stellar populations of 232 quiescent galaxies in the Shapley Supercluster, based on spectroscopy from the AAOmega spectrograph at the AAT. The key characteristic of this survey is its coverage of many low-luminosity objects $\left(\sigma \sim 50 \mathrm{~km} \mathrm{~s}^{-1}\right)$, with high signal-to-noise $\left(\sim 45 \AA^{-1}\right)$. Balmer-line age estimates are recovered with $\sim 25 \%$ precision even for the faintest sample members. We summarize the observations and absorption line data, and present correlations of derived ages and metallicities with mass and luminosity. We highlight the strong correlation between age and $\alpha$-element abundance ratio, and the anti-correlation of age and metallicity at fixed mass, which is shown to extend into the low-luminosity regime.
\end{abstract}

\section{Introduction}

The evolution of galaxy populations, subsequent to the peak of cosmic star formation at $z \sim 2$, has been driven by a progressive extinguishing of activity as galaxies lose or exhaust their gas. Recent surveys of galaxies at redshifts up to $z \sim 1$ (e.g. Bell et al. 2004; Faber et al. 2007) reveal the corresponding growth in stellar mass on the "Red Sequence" of quiescent galaxies. A theme which emerges from such work is the "downsizing" trend, whereby star-formation appears to be shut off at high redshifts in the most massive galaxies, while lower-mass galaxies are "quenched" only at more recent epochs.

Evidence for downsizing is also found in cluster galaxy populations. Distant clusters are deficient in faint Red-Sequence members, relative to local clusters, suggesting that low-mass galaxies continued to form stars well after the giant ellipticals were quenched (e.g. De Lucia et al. 2007; Stott et al. 2007). There is also evolution in the typical mass of "post-starburst" (post-quenching?) galaxies, with more massive galaxies passing through this short-lived phase at higher redshifts than the low-mass galaxies. (Tran et al. 2003).

An alternative to studying galaxy populations at higher redshifts is to infer the quenching history from detailed spectroscopic analyses of today's Red Sequence galaxies. In particular, comparison of observed metal and Balmer line strengths against model predictions can yield estimates of stellar population "age", weighted towards the most recent star-formation episode. Until recently, high signal-to-noise $(\mathrm{S} / \mathrm{N})$ absorption-line data were limited to fairly small samples of galaxies, and generally to giant elliptical galaxies (e.g. Kuntschner et al. 2001). Samples based on much larger spectroscopic surveys (e.g. Nelan et al. 2005; Bernardi et al. 2006; Graves et al. 2007) generally do not have sufficient $\mathrm{S} / \mathrm{N}$ to measure ages for individual galaxies, except at the highest luminosities. Nonetheless, these surveys do recover an average trend of younger ages for galaxies with lower $\sigma$, which is the expected signature of downsizing in the low-redshift fossil record.

Downsizing implies that today's faint Red Sequence galaxies are of crucial importance to understanding the evolution of galaxy populations at $z<1$. Some early progress in measuring galaxy ages in the low-luminosity regime was made by Mobasher et al. (2001) and Caldwell, Rose \& Concannon (2003). Here, we present new results from a 
comprehensive spectroscopic survey of galaxies in the Shapley Supercluster $(z=0.045)$ using the AAOmega spectrograph at the Anglo-Australian Telescope. Our study is unique in its combination of wide luminosity baseline coverage, large sample size, and high $\mathrm{S} / \mathrm{N}$ maintained even at low luminosity.

Section 2 of this paper summarizes the material published as Smith, Lucey \& Hudson (2007a). Section 3 presents further analysis, to appear in subsequent papers (Smith, Lucey \& Hudson, 2007b,c, in preparation).

\section{AAOmega observations and the index- $\sigma$ relations}

In April 2006, we obtained spectra for a total of 416 galaxies in the Shapley Supercluster, forming a magnitude-limited $(R<18)$ sample from the photometric catalogue of the NOAO Fundamental Plane Survey (NFPS, Smith et al. 2004). The sample covers the central $40 \times 40 \operatorname{arcmin}^{2}$ region in each of the three clusters Abell 3556, Abell 3558 and Abell 3562. Two AAOmega fibre configurations were employed, with $\sim 8$ hours total integration for each configuration. The spectra cover the range $3700-5800 \AA$ at a resolution of $3.5 \AA \mathrm{FWHM}$ in the blue spectrograph, and 5800-7300 $\AA$ at $1.9 \AA \mathrm{FWHM}$ in the red arm. The median $\mathrm{S} / \mathrm{N}$ for faint galaxies $\left(\sigma<100 \mathrm{~km} \mathrm{~s}^{-1}\right.$ ) is $\sim 45$ per $\AA$, (at rest-frame 4400-5400 $\AA$ ). For galaxies with $\sigma>100 \mathrm{~km} \mathrm{~s}^{-1}$, the median $\mathrm{S} / \mathrm{N}$ is $\sim 90$ per $\AA$.

Redshifts and velocity dispersions were measured using standard cross-correlation methods. Emission line equivalent widths were measured after removing a best-fiting stellar continuum model. The Lick absorption line indices (Trager et al. 1998), were measured on the blue-arm spectra, and converted to the Lick system using resolution transformations based on model SSP spectra, to avoid smoothing the observed spectra.

Our stellar population analysis is restricted to supercluster members using the measured redshift, and to quiescent galaxies defined by their $\mathrm{H} \alpha$ emission, $\mathrm{EW}(\mathrm{H} \alpha)<0.5 \AA$, yielding a sample of 232 galaxies. Figure 1 shows the correlations of selected absorption line indices with velocity dispersion for this sample. Note that $\sim 30$ galaxies have measured velocity dispersions consistent with zero and are shown at an arbitrary $\sigma$ at the left of the figure. Index $-\sigma$ slopes are fitted to the $\sim 200$ galaxies with measured $\sigma$.

The slopes of the index $-\sigma$ relations can be used to infer the scaling relations followed by the stellar population parameters (e.g. Nelan et al. 2005; Smith et al. 2006). For the Thomas et al. (2003) models, the derived parameters are age, total metallicity, $[\mathrm{Z} / \mathrm{H}]$ and $\alpha$-element abundance ratio, $[\alpha / \mathrm{Fe}]$, estimated by comparison to predictions for simple stellar populations (SSPs). Fitting for the slopes of nine index $-\sigma$ relations, in comparison to the these models, we recover: Age $\propto \sigma^{0.52 \pm 0.10}, \mathrm{Z} / \mathrm{H} \propto \sigma^{0.34 \pm 0.07}$, and $\alpha / \mathrm{Fe} \propto$ $\sigma^{0.23 \pm 0.06}$. The quoted errors include a (dominant) contribution from sytematic errors, estimated using a large set of different indices in the fitting process.

The age- $\sigma$ scaling relation is consistent with that obtained by Nelan et al. (2005) from similar analysis of the NFPS, and implies that even if the most massive red galaxies formed at very high redshift, much of the faint red population became quiescent only at recent epochs $(z<0.5)$. As shown in Smith et al. (2007a), the implied deficit of faint red galaxies as a function of redshift agrees quantitatively with the observed evolution in the Red Sequence luminosity function (Stott et al. 2007; De Lucia et al. 2007).

\section{The age-metallicity-mass relations}

To move beyond the average scaling relations, we use stellar population models to determine the values of age, $[\mathrm{Z} / \mathrm{H}]$ and $[\alpha / \mathrm{Fe}]$ which reproduce a non-degenerate set of observed indices, for each galaxy individually. The results shown here are derived from fitting to the models of Thomas et al. (2003), using indices $\mathrm{H} \gamma_{\mathrm{F}}$, Fe5015 and Mgb5177. 

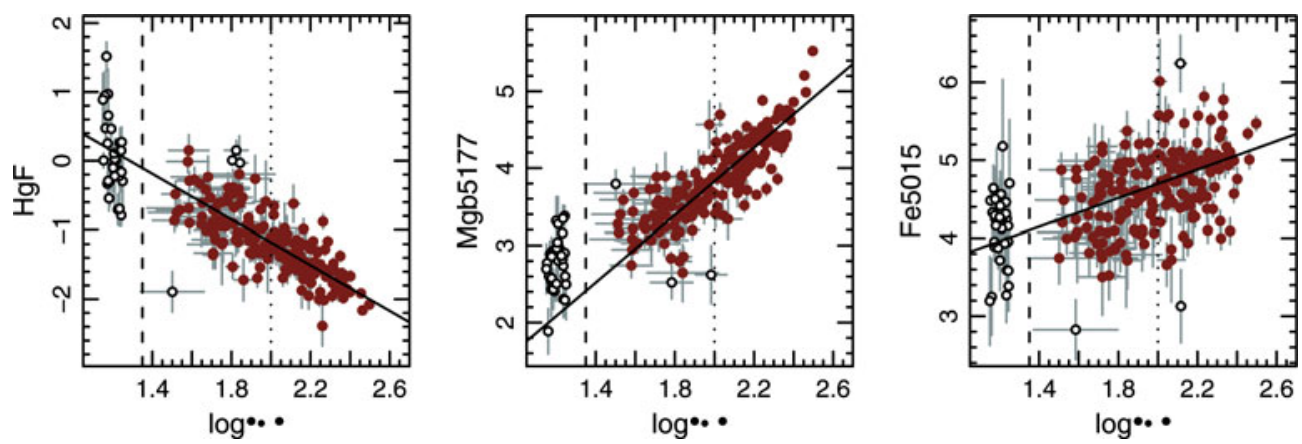

Figure 1. The index- $\sigma$ relations for selected indices. Objects to the left of the dashed line have velocity dispersions consistent with zero, and are not used in fitting the relations. Open symbols represent these and also other galaxies excluded from the fit by an iterative outlier rejection. The dotted line at $\sigma=100 \mathrm{~km} \mathrm{~s}^{-1}$ shows the typical low-mass limit of many other studies.
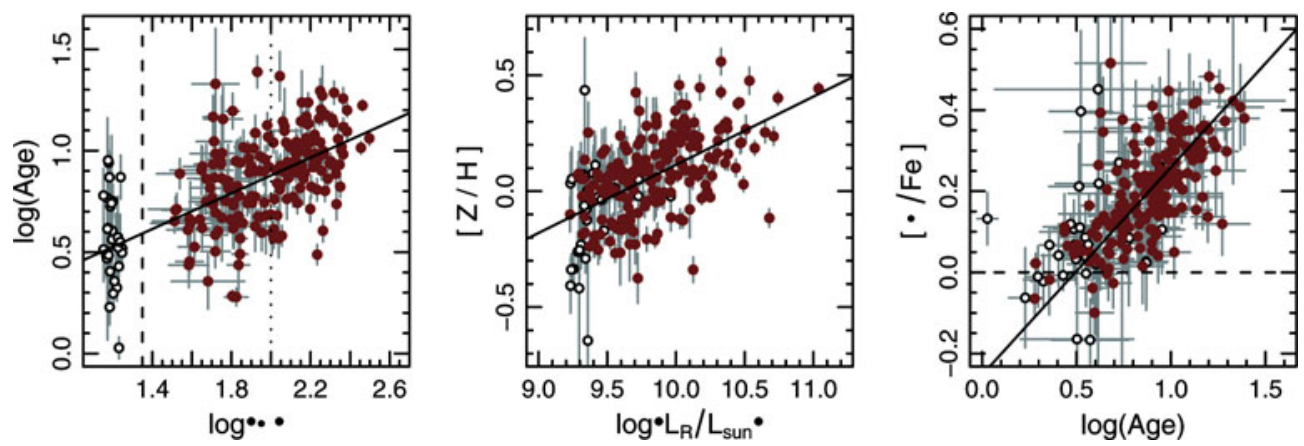

Figure 2. Left: Ages versus velocity dispersion. Centre: total metallicity versus luminosity. Right: $[\alpha / \mathrm{Fe}]$ versus age. The SSP-equivalent parameters were derived by comparison to models of Thomas et al. (2003). Galaxies with unresolved velocity dispersions are shown by open symbols and placed at arbitrary position in the left panel.

For the faintest galaxies in the sample $\left(L_{R} \sim 10^{9.5} L_{\odot}\right)$, the median formal errors are $25 \%$ in age, 0.08 dex in $\mathrm{Z} / \mathrm{H}$ and $\alpha / \mathrm{Fe}$. Some key correlations of the derived parameters are shown in Figure 2. Intriguingly, we find that although age is correlated more tightly with $\sigma$ than with luminosity, metallicity is better correlated with luminosity. The abundance ratio $[\alpha / \mathrm{Fe}]$ is well-correlated with $\sigma$ and also follows a tight age $-[\alpha / \mathrm{Fe}]$ relation. The latter suggests that "younger" galaxies formed stars over more extended periods, broadly consistent with a quenching scenario rather than formation in a single rapid burst.

Figure 3 shows the relationship between age and metallicity for three intervals in $\sigma$. As expected from the previous figure, both age and $[\mathrm{Z} / \mathrm{H}]$ increase with increasing $\sigma$, on average. However, at fixed $\sigma$, they are anti-correlated: galaxies which are younger than average for their mass are also more metal-rich than average. The sample populates a plane (the "Z-plane") described by $[\mathrm{Z} / \mathrm{H}]=0.66 \pm 0.04 \log \sigma-0.68 \pm 0.04 \log$ Age $-0.64 \pm$ 0.08 , with scatter $\sim 0.1$ dex in $[\mathrm{Z} / \mathrm{H}]$. The coefficients are consistent with those obtained by Trager et al. (2000) for a smaller sample, mostly comprising giant elliptical galaxies. Our results demonstrate the continuity of this plane into the low-luminosity regime.

The age coefficient in the Z-plane is such that the distribution of galaxies is approximately aligned with the age-metallicity degeneracy, so that the reddening effect of higher metallicity compensates for the bluer colours expected of younger populations. The tightness of the tight colour-magnitude sequence is a partly consequence of this "conspiracy". 

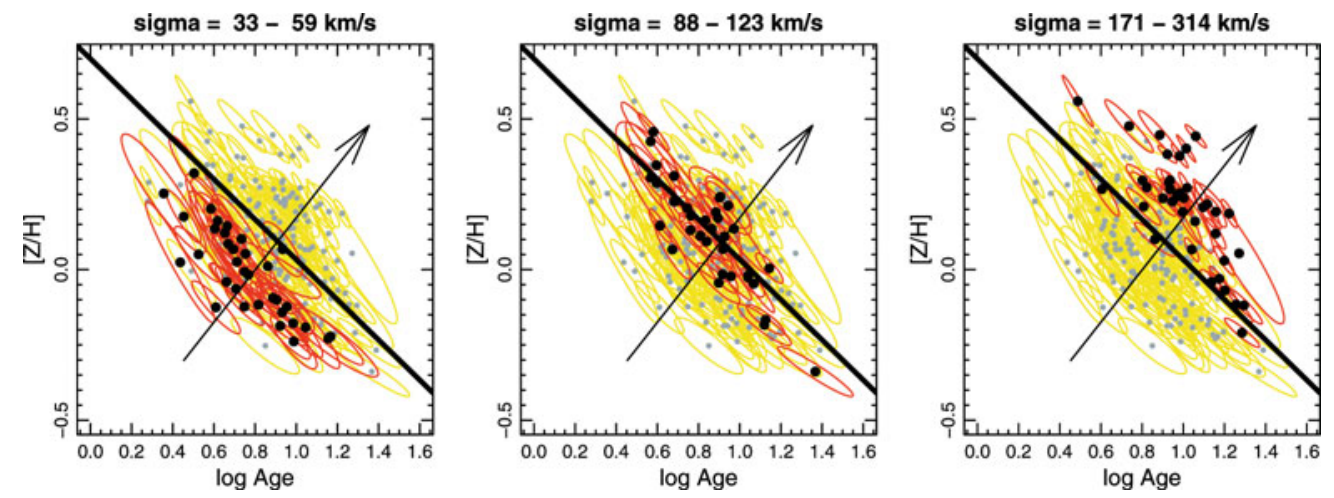

Figure 3. The Age-Metallicity-Mass relations. The black points and red ellipses show the estimated and $1 \sigma$ confidence intervals for galaxies in each of three intervals in velocity dispersion. The rest of the sample is shown in grey/yellow for reference. The arrow indicates the direction in which the median age and metallicity move with increasing mass. The heavy black line (same in all panels) indicates the direction of the age-metallicity degeneracy: movement parallel to this line generates no change in galaxy broadband colours.

Note that although the error ellipses are oriented parallel to the intrinsic dispersion, the measurement errors can account for only $\sim 20 \%$ of the observed variance along the plane.

The existence and slope of the age-metallicity anti-correlation at fixed mass is crucial to understanding the star-formation history of the galaxies prior to quenching. In Smith, Lucey \& Hudson (2007c, in preparation), we describe a simple self-enrichment model for the pre-cursors of today's Red Sequence galaxies, and show that the rate of metallicity growth required to fit the Z-plane agrees quantitatively with the evolution observed in the metallicity-mass relation for star-forming field galaxies (e.g. from Erb et al. 2006).

\section{Acknowledgements}

RJS is supported under the STFC rolling grant PP/C501568/1 "Extragalactic Astronomy and Cosmology at Durham 2005-2010".

\section{References}

Bell, E. F., et al. 2004, ApJ, 608, 752

Bernardi, M., Nichol, R. C., Sheth, R. K., Miller, C. J., \& Brinkmann, J. 2006, AJ, 131, 1288

Caldwell, N., Rose, J. A., \& Concannon, K. D. 2003, AJ, 125, 2891

De Lucia, G., et al. 2007, MNRAS, 374, 809

Erb, D. K., Shapley, A. E., Pettini, M., et al. 2006, ApJ, 644, 813

Faber, S. M., et al. 2007, ApJ, 665, 265

Graves, G. J., Faber, S. M., Schiavon, R. P., \& Yan, R. 2007, ApJ, in press, arXiv:0707.1523

Kuntschner, K., Lucey, J. R., Smith, R. J., et al. 2001, MNRAS, 323, 615

Mobasher, B., et al. 2001, ApJS, 137, 279

Nelan, J. E., Smith, R. J., Hudson, M. J., et al. 2005, ApJ, 632, 137

Smith, R. J., et al. 2004, AJ, 128, 1558

Smith, R. J., Hudson, M. J., Lucey, J. R., et al. 2006, MNRAS, 369, 1419

Smith, R. J., Lucey, J. R., \& Hudson, M. J. 2007a, MNRAS, in press, arXiv:0707.1695

Stott, J. P., Smail, I., Edge, A. C., et al. 2007, ApJ, 661, 95

Thomas, D., Maraston, C., \& Bender, R. 2003, MNRAS, 339, 897

Trager, S. C., Faber, S. M., Worthey, G., \& González, J. J. 2000, AJ, 120, 165

Tran, K.-V. H., Franx, M., Illingworth, et al. 2003, ApJ, 599, 865 